\title{
REGARDS JOURNALISTIQUES SUR LE TRAVAIL DES RÉDACTIONS EN LIGNE
}

\begin{abstract}
Amandine Degand $^{1}$
Cet article aborde la question des représentations journalistiques envers le journalisme en ligne, les rédactions Internet et les charges qui leur incombent. De précédentes recherches ont esquissé les traits d'un malaise envers le webjournalisme, d'une déconsidération, voire d'un mépris envers les équipes en ligne (Friend \& Singer, 2007 ; Estienne, 2007 ; Quandt, 2008). Mais qu'est-ce qui déplaît tant ? Et à l'inverse, quels sont les aspects appréciables que les journalistes attribuent au webjournalisme tel qu'il est pratiqué dans leur entreprise?

Pour répondre à ces questions, nous prendrons appui sur une enquête de type ethnographique : 56 jours d'immersion dans 11 rédactions belges et 101 entretiens semi-directifs auxquels nous avons appliqué une analyse thématique au moyen du logiciel Nvivo (Paillé \& Mucchielli, 2003). Grâce à ce vaste matériel empirique, nous examinerons les opinions, positives et négatives, que les journalistes entretiennent à l'égard du webjournalisme. L'un des résultats les plus marquants de cette enquête est l'omniprésence de l'activité (auto-)critique des journalistes. Cette critique, en étant continuellement temporisée par des arguments plus positifs, apparaît comme l'un des nœuds permettant, dans un processus dialogique (Le Cam, 2010), la perpétuelle recomposition de l'identité professionnelle.
\end{abstract}

1 Amandine Degand est Chercheuse à l'Université catholique de Louvain.

Recherches en communication, $\mathrm{n}^{\circ} 39$ (2013). 
Dans une approche compréhensive, cet article s'intéresse aux représentations à l'égard du webjournalisme et de sa pratique. Plus précisément, nous nous focaliserons sur jugements positifs et négatifs énoncés par les journalistes - que ces derniers travaillent ou non sur ce support - au sujet du webjournalisme tel qu'il est pratiqué dans une rédaction donnée.

Notre enquête repose sur 56 jours d'immersion dans 11 rédactions de Belgique francophone ${ }^{1}$. Cette observation, réalisée entre la fin 2009 et le début 2010, nous a notamment permis de recueillir 101 entretiens de journalistes et de professionnels de l'information. A ce matériel empirique, nous avons appliqué une analyse thématique, couplée à une analyse par catégories conceptualisantes (Paillé \& Mucchielli, 2003) au moyen du logiciel Nvivo. Nous avons enfin actualisé nos données par 10 entretiens téléphoniques ${ }^{2}$, en janvier 2012.

\section{État de l'art}

Les premières représentations à l'égard du journalisme Internet étaient plutôt positives, voire franchement utopistes. A la fin des années 1990, les journalistes prennent lentement conscience $\mathrm{du}$ formidable potentiel d'Internet en tant que source d'information. La recherche présentait alors majoritairement ce 'cyberjournalisme' comme une voie pour améliorer la couverture de l'actualité (Pavlik \& Ross, 2000, cités par Le Cam, 2005).

Mais, parallèlement, des études mettent en avant des dérives professionnelles associée à l'émergence du webjournalisme telles que l'intensification des pressions commerciales et hiérarchiques qui pèsent sur les journalistes ou encore la précarisation des journalistes et l'alourdissement des charges qui leur incombent (Deleu et al., 1998). D'aucuns estiment que l'arrivée d'Internet favorise l'émergence d'un journaliste expert en traitement d'info, voire d'un simple fournisseur

1 Le Soir (Quotidien généraliste) - La Libre Belgique/La Dernière Heure (Quotidien généraliste/ Quotidien spécialisé dans le sport et les faits divers. Groupe IPM) Les Editions de l'Avenir (Groupe de quotidien locaux) - Sudpresse (Groupe de quotidiens locaux) - Le Vif/L'Express (Hebdomadaire) - 7 sur 7 (Site pure player) $L^{\prime} E c h o$ (Quotidien économique) - RTBF (Entreprise audiovisuelle publique) - RTL (Entreprise audiovisuelle privée) - Belga (Agence de presse) - BFM Today (Radio économique reconvertie en pure player, aujourd'hui disparue).

2 Nous avons recontacté par téléphone chacun des responsables des cellules web visitées, à l'exclusion de BFM Today, qui, entre temps, a mis clef sous porte. 
d'informations. Ce phénomène renverrait à un traitement de type «techno-bureaucratico-commercial » de l'information (Pélissier, 2003, p. 105). Pour Deni Elliott, la contrainte de production d'information en continu, dans un univers hyperconcurrent, conduit les journalistes à manquer de rigueur dans leur travail (cité par Bernier, 2008, p. 24). C'est ce qui pousse certains chercheurs à affirmer que ce contexte de production n'offre pas les ressources nécessaires pour un journalisme indépendant, soucieux de l'intérêt public (Fenton \& Witschge, 2011, p. 159). Pour Boczkowski (2009), les technologies avec lesquelles travaillent désormais les journalistes les conduisent de plus en plus à surveiller et imiter leur concurrence. Son étude

shows how changes in information gathering (the increasing use of scopic infrastructures of mediation and the intensification of monitoring) have been tied to alterations in meaning making (the expansion of imitation resulting from the growing conformity to peer's judgments of newsworthiness), all of which have diminished the critical function of journalists to foster diversity of ideas in the public sphere $(2009$, p. 55).

D'aucuns estiment en outre que les nouvelles technologies contribuent à banaliser et à simplifier le travail du journaliste. De ce fait, le pouvoir et le prestige social de cette profession s'en trouvent diminués (Deuze \& Fortunati, 2011, p. 169). Les journalistes, s'éloignant des tâches traditionnelles du reportage, pourraient se transformer en passifs réceptacles d'information (Kovach \& Rosenstiel, 2001, p. 76).

C'est sans doute dans ce contexte de craintes quant à l'avenir de la profession qu'à mûrit une représentation négative des équipes Web, au sein même de leur espace professionnel. De nombreuses recherches ont montré que les journalistes Web sont officieusement perçus par leurs collègues comme des journalistes de seconde zone (Garcia, 2008, p. 72) ou de seconde main (Quandt, 2008, p. 89).

Ceux qui côtoient aujourd'hui les rédactions de près pourront objecter que ce cliché, s'il n'est pas dépassé, a au moins évolué vers une représentation nettement plus positive. A mesure que des initiatives originales se développent en ligne (notamment grâce aux avant-gardes du datajournalism, du cross-media, du journalisme participatif ou encore du webdocumentaire), les journalistes Web redorent progressivement leur blason. Nous pensons néanmoins que les rédactions resteront durablement marquées par ces déconsidérations, 
aussi éphémères fussent-elles. En effet, si les journalistes Web parviennent, petit à petit à légitimer leur rôle et leur professionnalisme, il n'en reste pas moins que l'émergence du journalisme numérique a introduit dans les rédactions des stratégies de convergence et des réorganisations qui ont un impact sur le bien être des journalistes, sur leur représentation de rôle, et, pour une frange grandissante de la population journalistique, sur leurs pratiques quotidiennes.

L'étude de Jane B. Singer (2004) a par exemple montré que la convergence complique considérablement le travail des journalistes. Néanmoins, aucun d'entre eux n'est allé jusqu'à prétendre que la convergence mène à un journalisme de qualité médiocre. L'auteur synthétise, par ailleurs, quelques unes des critiques formulées à l'égard des modes d'organisation convergents :

One key concern is the potential for fewer journalists to be hired and thus fewer reporters to perform watchdog and other democratic roles. Another has been that when managers ask journalists to do multiple tasks, including some for which they have little or no expertise, the result will be a mediocre product that fails to meet its public service obligations (Friend \& Singer, 2007, pp. 201-202).

Dans une étude belge, C. Fion (2008) avait également noté que pour $54 \%$ des journalistes interrogés, la réalisation d'un travail multimédia (ou multi-support) représentait une menace pour la qualité de l'information. Enfin, Josep Micó et ses collègues (2009) indiquent que la convergence entraine notamment une standardisation du discours, une perte de pluralisme, un déclin de la qualité du journalisme, une réduction des effectifs de la rédaction et une augmentation des charges de travail.

A l'inverse, des études ont mis en avant les aspects positifs liés au webjournalisme et à sa pratique. Le travail multi-tâches est par exemple décrit par les professionnels comme une opportunité, une ouverture vers de nouveaux postes pour les jeunes journalistes (Carvajal \& García Aviles, 2008, p. 231). Jane B. Singer arrive à la même conclusion auprès des journalistes du média pionnier en matière de convergence, l'américain Tampa Bay Online (TBO) : la capacité de travailler pour différents médias y est interprété comme un atout sur le curriculum vitae (2004a). Edgar Huang et ses collègues (2004) estiment que la qualité du journalisme se dégrade précisément parce 
que la convergence n'est pas le mode d'organisation dominant. Dans leur étude de The Tampa Tribune, ils constatent qu'une organisation convergente permet de maintenir un journalisme de qualité. T. Bettels (2005) constate que, dans les rédactions de Main Post (Allemagne) et de Nordjykse Medier (Danemark), $60 \%$ des journalistes interrogés estiment que la qualité de leur journalisme s'est améliorée avec l'installation d'une organisation convergente. La satisfaction au travail a également augmenté significativement. M.-F. Bernier(2008) précise que le mode de travail convergent n'est pas forcément nuisible pour le journalisme, mais il faut assurer l'autonomie des journalistes et limiter les appétits financiers des actionnaires de presse.

Certains auteurs se sont également intéressés aux profils des journalistes les plus critiques ou les plus positifs face au webjournalisme et aux modes organisationnels qui y sont associés. Ainsi, une étude a constaté que c'étaient généralement les journalistes dans la situation la moins favorable - les webjournalistes le plus souvent - qui défendaient l'intégration, alors que les journalistes mieux établis craignaient de voir leurs salaires diminuer à cause de l'intégration (Domingo et al., 2008, cités par Micó et al., 2009). Ivar John Erdal, pour sa part, classe les journalistes de $N R K$ - l'opérateur public norvégien - en trois groupes, selon leurs représentations par rapport au travail cross-média : l'un positif, le second négatif et le troisième positif en théorie, mais réticent en pratique. Au sein du groupe négatif, l'argument phare est que la collaboration entre médias pourrait nuire à la qualité du journalisme et anéantir la réputation (legacy) de $N R K$ (2009, p. 223). Ceci provient sans doute du fait que les journalistes se représenteraient toujours leurs collègues issus des autres supports médias comme étant moins respectueux des normes éthiques qu'eux-mêmes (Friend \& Singer, 2007, p. 207).

\section{Le contexte belge}

$\mathrm{Vu}$ la difficulté, pour les journalistes, de construire une représentation positive du webjournalisme, plusieurs responsables multimédia que nous avons interrogé dans plusieurs rédactions belges ont exprimé leur souhait de ne pas forcer les collaborations entre les différents segments professionnels - dans un premier temps -, mais de les encourager. Dans cette perspective, les collaborations entre segments professionnels doivent idéalement devenir 'naturelles'. Pour ce faire, différentes stratégies peuvent être mises en place. Des 
systèmes d'édition (CMS) communs pour le Web et le papier sont créés. Les réunions de rédactions des médias traditionnels accueillent progressivement un représentant du Web. Un bureau central circulaire (ou table de convergence) est parfois installé au centre de la salle de rédaction 'historique' : il doit marquer, dans le paysage, la nécessité nouvelle de distiller une même information au prisme des différents canaux disponibles (médias 'traditionnels', Web, réseaux sociaux, mobiles, tablettes, etc.). Il permet en outre de placer, symboliquement, des journalistes Web au centre de la rédaction. Certains médias vont jusqu'à bouleverser considérablement l'organisation du travail. C'est le cas de $L ' E c h o$, où est instaurée une rotation à l'édition du site Internet : l'ensemble des journalistes de la rédaction passe quelques jours par mois aux postes 'Web'.

A l'heure de nos interviews, ces stratégies sont supposées porter leurs fruits. Mais force est de constater que la critique est toujours cinglante. Les représentations les plus négatives proviennent des journalistes qui n'ont que peu de liens avec les équipes Web. Pour la plupart, ils marquent, dans leurs discours, une distance, voire une rupture par rapport aux équipes Web. Sur le terrain observé, ils sont encore nombreux ces journalistes dont le travail reste essentiellement 'traditionnel' : la plupart du temps, ils peuvent se contenter de travailler pour leur média premier (la presse imprimée, la radio ou la télévision). Lors de nos immersions en rédactions, la césure entre le Web et le nonWeb est encore assez nette, dans les discours d'acteurs comme dans les faits. Cela dit, dans les représentations, les différences sont plus ténues. De manière assez surprenante, les journalistes Web eux-mêmes marquent une distance par rapport à leur propre occupation. S'ils tentent de mettre certaines de leurs qualités professionnelles en valeur, n'en sont pas moins critiques par rapport à leur propre pratique.

\section{Les opinions sur le webjournalisme et sa pratique}

Lors de nos interviews, plusieurs jugements à l'égard du webjournalisme ont été énoncés. Nous les avons classés comme positifs si les journalistes exprimaient des caractéristiques motivantes ou appréciables du webjournalisme. Nous les avons classés comme négatifs si les journalistes faisaient état de traits dérangeants, démotivants ou dommageables dans le webjournalisme et sa pratique. Dans les tableaux ci-dessous, nous avons dénombré les différents arguments développés dans chacune des deux catégories. Ce décompte n'a aucune valeur 
statistique puisque les réponses n'ont pas été recueillies dans le cadre de questionnaires standardisés. Mais il permet de se faire une idée du poids respectifs des différents arguments, certains étant beaucoup plus souvent cités spontanément par les répondants.

\section{Les discours positifs sur le webjournalisme et sa pratique}

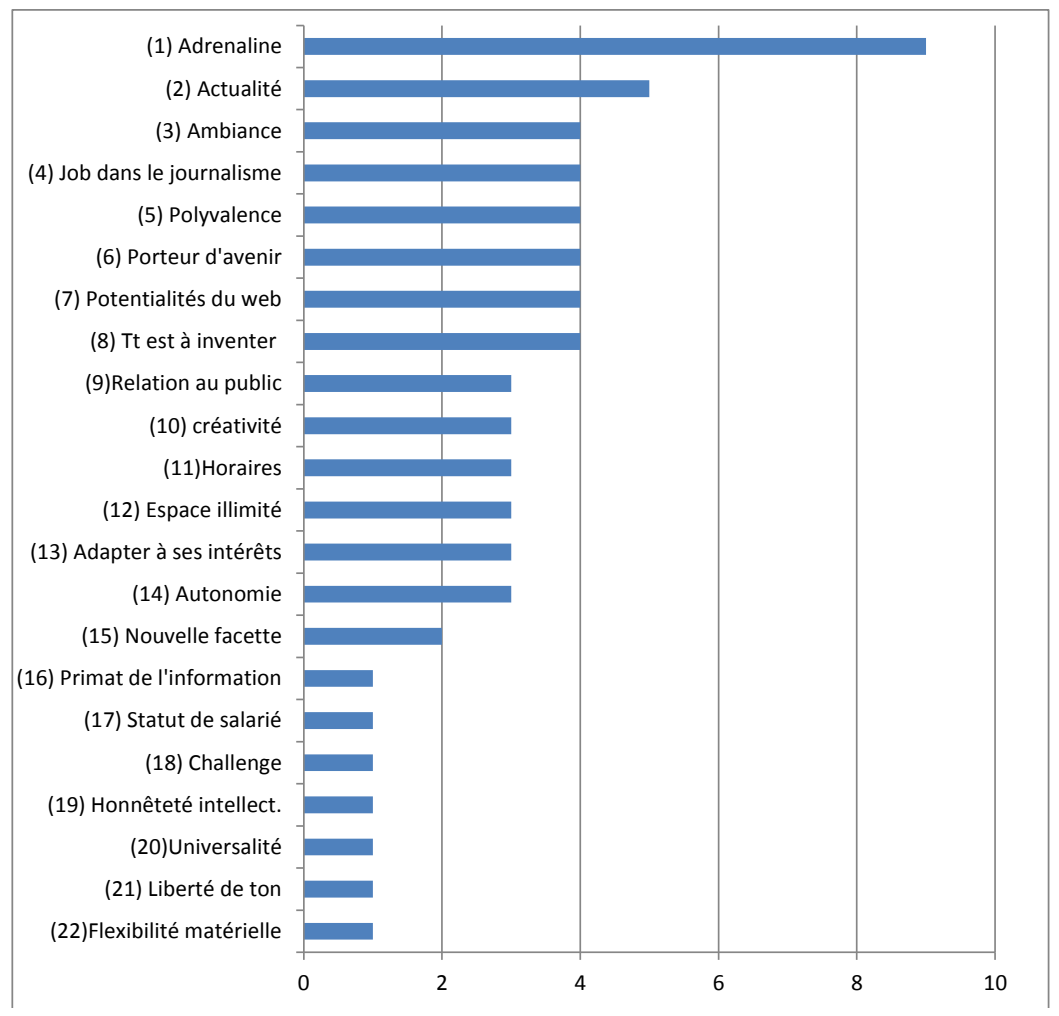

Figure 1. Nombre de journalistes ayant cité chacun cité l'un des aspects positifs du webjournalisme (réponses codées dans le sous-thème 'Motivant-Appréciable' suite à l'analyse thématique réalisée avec le logiciel Nvivo 8).

L'aspect du webjournalisme le plus souvent cité comme appréciable lors des entretiens est (1) l'adrénaline, la 'prise de risque' ou le 'stress enivrant' que les journalistes éprouvent soit quand ils travaillent de façon très réactive, dans l'immédiateté, soit lorsqu'ils réalisent un travail multi-support intense pour la couverture d'une actualité donnée. Ce premier aspect appréciable se détache nettement des autres, avec neuf mentions. 
Le second élément de réponse, mentionné par 5 journalistes, est le contact avec l'actualité chaude (2), le fait d'être toujours au courant de tout. Quatre autres considèrent ensuite que les petites équipes de travail telles que l'on peut les trouver dans les rédactions Web sont de nature à fournir une ambiance de travail appréciable (3). Quatre webjournalistes s'estiment déjà bien heureux d'avoir un emploi, un job dans le journalisme (4). Les journalistes apprécient également la polyvalence (5) que peut leur apporter un emploi sur le Net et le fait de travailler pour un projet qu'ils perçoivent comme étant 'porteur d'avenir' (6). Plusieurs journalistes apprécient pouvoir profiter des nombreuses potentialités du Web (7) : du chat au postcast en passant par la possibilité de réaliser des animations flash ou des vidéos. Ils sont également nombreux à apprécier le fait que 'tout est à inventer' (8) dans l'univers de l'information en ligne.

Parmi les caractères appréciables cités par trois journalistes, figurent la relation qu'ils entretiennent avec le public d'internautes via les logiciels de mesure d'audience ou les espaces interactifs (9), l'aspect créatif du travail en ligne (10), les horaires de travail (11), le fait que, parce que l'espace virtuel est illimité, le webjournaliste peut fournir des contenus allant au-delà des formats très stricts imposés dans l'audiovisuel ou la presse imprimée (12), la possibilité d'adapter leurs occupations professionnelles à leurs intérêts, grâce à une plus grande liberté de choix des sujets à traiter (13) et l'autonomie qui leur est accordée quand ils travaillent à l'édition d'un site Web dans sa globalité (14). Deux personnes ont indiqué que le webjournalisme en ligne apporte un changement, bienvenu dans une carrière professionnelle, qu'il permet de découvrir une nouvelle facette du métier (15).

Enfin, les caractères appréciables suivants n'ont été mentionnés que par un journaliste. Cela ne signifie pas forcément que ces opinions ne sont pas partagées par d'autres personnes au sein de la population interrogées, mais plutôt qu'elles sont moins ancrées dans les représentations, moins généralisées et donc moins souvent abordées. Ainsi, une journaliste, qui a travaillé aux débuts du Net et qui revient rétrospectivement sur son expérience dit qu'elle aimait le fait qu'il n'y avait pas de 'coups médiatiques', que l'information primait sur toute autre considération (16). Un autre dit apprécier le fait qu'il bénéficie d'un statut de salarié sur le Net (17). Un journaliste, parce qu'il est issu de $7 \operatorname{sur} 7$, média qui n'a pas de véritable équivalent papier 
historique, dit apprécier le challenge qui constitue à imposer un titre Web dans un monde médiatique préétabli (18). Citons enfin l'honnêteté intellectuelle associée au support Internet qui, souvent, cite ses sources (19), l'universalité du support consultable dans le monde entier (20), la liberté de ton possible dans l'écriture sur le Web (21) ou la flexibilité matérielle rendue possible par l'Internet qui permet, avec peu de moyens, de poster un article en ligne depuis n'importe quel lieu (22).

\section{Les discours négatifs sur le webjournalisme et sa pratique}

Le second graphique illustre quelques pics très nets qui correspondent à des aspects particulièrement dérangeants ou déplaisants dans le webjournalisme, pics qui n'ont pas d'équivalents dans le graphique précédent.

Non moins de 23 répondants dénoncent le manque de moyens (humains, financiers, temporels, etc.) dont souffrent les équipes du Net (1). Le second reproche fait au webjournalisme porte sur sa tendance au sensationnalisme, à la sélection d'information réalisée davantage sur des critères marketing que sur des critères journalistiques. Ils sont 16 à considérer qu'il y a là une dérive, potentielle ou avérée (2). Troisième défaut du webjournalisme le plus souvent mentionné par les journalistes, le manque de vérification de l'information reflète également une inquiétude de la part des journalistes face à un déclin de la crédibilité de la presse (3). Quatrième élément déplaisant le plus cité, la gestion de l'interactivité remporte 10 mentions (4). C'est avant tout le manque d'encadrement des fils de discussion qui est pointés. Neuf journalistes ont relevé le manque de travail de terrain qui est également l'un des éléments les plus critiqués par les répondants lorsqu'ils jugent le webjournalisme (5).

Passons ensuite dans un registre d'opinions qui, si elles sont toujours citées par six à trois personnes, ne sont plus aussi généralisées que les représentations précédentes. Ainsi, six journalistes déplorent la multiplication des tâches qui incombent à une seule personne et le travail multi-support (6). Ces nouvelles logiques de travail conduisent, selon plusieurs journalistes interrogés, à la réalisation d'un travail médiocre. Cinq journalistes Web déplorent la redondance de leurs tâches, le manque de diversité dans leur travail (7). Leur emploi est en effet décrit comme répétitif, ennuyeux et trop technique. Le suivisme (8) exaspère également quatre répondants. C'est avant tout le manque de réflexion est qui mis en cause, quand un média reprend aveuglément 
une information, simplement parce qu'il l'a vue ailleurs. Pour quatre journalistes interrogés, le plagiat (9) des productions de la concurrence est un aspect dérangeant de la pratique du webjournalisme. «Le copiécollé... c'est une plaie » (Journaliste Web, RTBF, Août 2009).

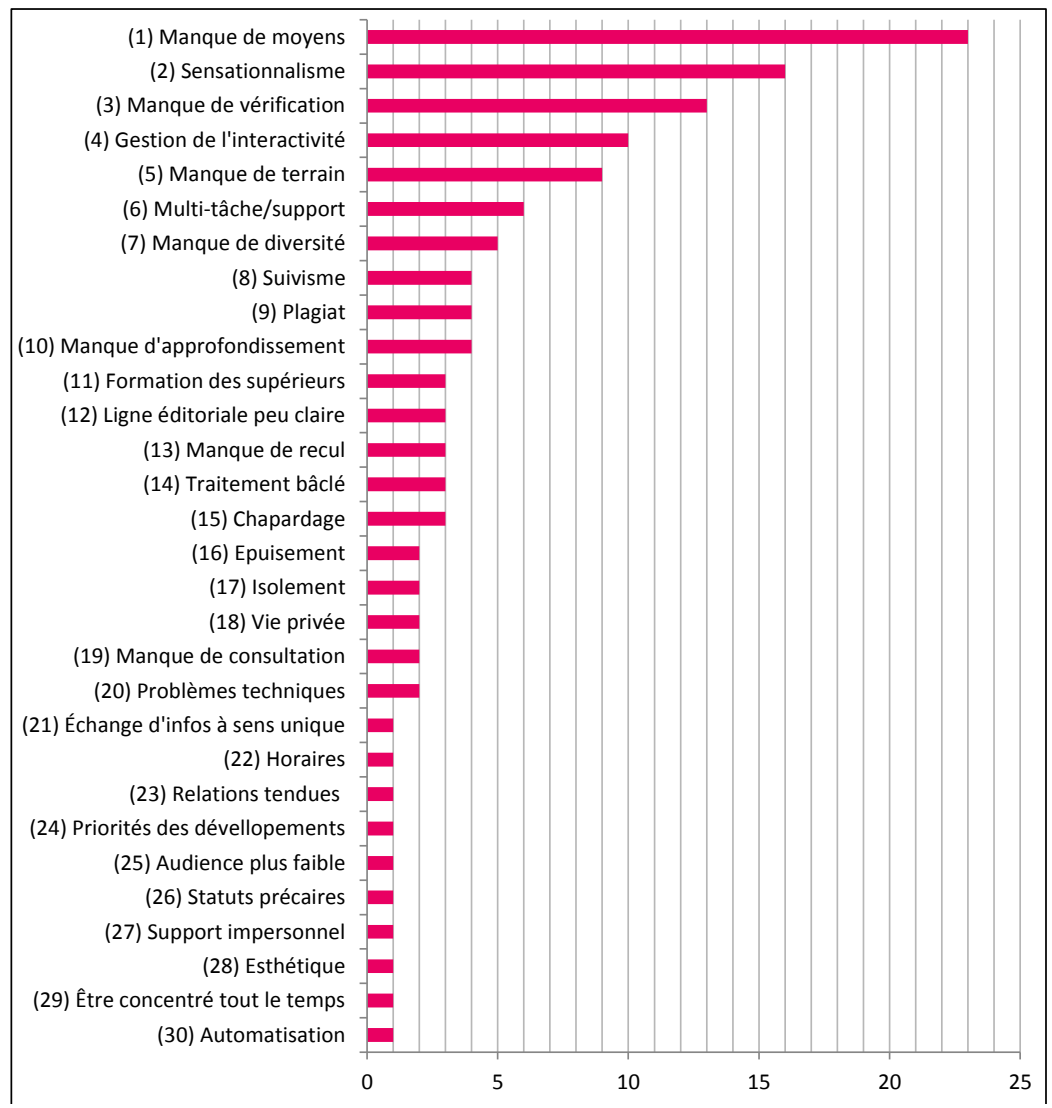

Figure 2. Nombre de journalistes ayant cité chacun des aspects négatifs du webjournalisme (réponses codées dans le sous-thème 'Dérangeant - Démotivant Dommageable' suite à l'analyse thématique réalisée avec le logiciel Nvivo 8).

Quatre webjournalistes se plaignent d'un manque d'approfondissement des sujets (10). «Ici, [du fait] d'être généraliste, tu n'approfondis jamais rien » (Journaliste Web, Le Soir, Juin 2009). Un autre type de reproche, partagé par trois répondants, s'adresse à la formation des supérieurs, des responsables des équipes Web (11) : 
les journalistes désapprouvent en effet le fait d'être sous l'autorité de personnes qui ont plus de liens avec les pôles marketing qu'avec les pôles rédactionnels. Trois journalistes traditionnels regrettent l'absence d'une ligne éditoriale claire pour le site Web (12). Ils estiment que ceci peut créer des tensions entre les différents supports d'une même marque média. Trois autres (Web ou non-Web) reprochent également le manque de recul (13) dont bénéficient les webjournalistes par rapport à l'information qu'ils doivent traiter. Un webjournaliste se plaint par exemple de ne pas avoir le temps de lire les journaux le matin. C'est également le manque de temps qui est jugé responsable des traitements bâclés de l'information, dont l'incarnation la plus visible se trouve dans les fautes d'orthographe (14).

$\mathrm{Au}$ rang des opinions les moins fréquemment relevées, figurent le manque de fair-play des médias quand il s'agit de citer sa concurrence, le chapardage de contenus dont les auteurs ne sont pas cités (15), l'aspect épuisant ou fatigant d'un travail à flux tendu (16), l'isolement et le sentiment de solitude des journalistes qui, soit travaillent en horaires décalés, soit se sentent trop peu nombreux face à des tâches multiples (17). Citons encore une intrusion du travail dans la vie privée (18) : deux journalistes Web disent travailler chez eux, en dehors de leurs horaires. Deux autres regrettent de ne pas être davantage consultés sur les questions liées à l'adoption de certaines innovations ou stratégies (19) ou se plaignent de problèmes techniques récurrents qui tardent à être réglées (20).

Dix aspects négatifs n'ont été cités que par un seul répondant : le fait que les collaborations soient à sens unique, que ce soit toujours aux journalistes traditionnels que l'on demande d'apporter des contenus pour le site, et non l'inverse (21), les horaires de travail qui semblent difficile aux yeux d'un journaliste traditionnel (22), les relations tendues voire agressives au sein de la rédaction Web (23), la priorité accordée à certains développements techniques, comme les applications Facebook sur la page d'accueil des sites, au détriment d'autres (24), le fait de ne pas se sentir valorisé de travaillé pour un support qui a une plus audience faible (que celle de la télévision) (25), les statuts précaires des webjournalistes (26), le caractère impersonnel du support Web, l'anonymat des journalistes qui y travaillent(27), le manque d'esthétique du site Web de son média (28), la nécessité d'être concentrée durant de longues périodes lors de l'édition du site (29) ou, enfin, les problèmes associés à l'automatisation de la mise en ligne 
de certains contenus qui engendre des erreurs et des tâches techniques pesantes (30).

\section{Discussion}

\section{Omniprésence de la critique}

Premier élément frappant, les aspects du Web perçus comme déplaisants (96 citations relevées et regroupées en 30 catégories de réponse) sont numériquement supérieurs aux aspects jugés plaisants (60 citations relevées et regroupées en 22 catégories de réponse). Ceci corrobore nos précédents constats empiriques mettant en lumière l'importance de la critique dans les discours journalistiques étudiés. Nous avions notamment relevé, d'une part, une forme de critique exogène, issue des journalistes non-Web (des représentations essentiellement négatives des équipes Web, une attitude de frilosité face aux demandes de collaboration avec les équipes Web) et, d'autre part, une critique endogène, ou auto-critique de la part des journalistes en ligne (une posture de distanciation face aux pratiques Webjournalistiques, un manque de vocation pour le poste occupé ou encore le peu d'enthousiasme et d'ambitions de carrière dans les médias en ligne) (Degand, 2012, p. 502). A priori, il parait donc normal de trouver, dans nos comptages descriptifs, un plus large éventail d'aspects négatifs énoncés envers le webjournalisme.

Les cinq principales ${ }^{1}$ critiques énoncées àl'égard du webjournalisme sont toutes associées à une volonté de voir le travail réalisé d'une manière plus qualitative. Elles mobilisent, par là même, un certain idéal journalistique que les répondants ont intégré. Á l'inverse, parmi les critiques les moins citées ${ }^{2}$, figurent nombre de considérations relatives au confort personnel des journalistes. Ces critiques, parce qu'elles sont marginales par rapport aux autres, donnent à penser que les journalistes seraient prêts à passer outre les petits désagréments associés à la pratique du webjournalisme, pour autant que leur métier réponde, par ailleurs, à leurs aspirations profondes.

Les critiques que les journalistes formulent aujourd'hui à l'égard du webjournalisme ne sont pas sans rappeler celles qui ont, de tout

1 Manque de moyens, sensationnalisme, manque de vérification, gestion de l'interactivité, manque de travail de terrain (voir second tableau).

2 Epuisement, isolement, relations tendues, horaires, intrusion dans la vie privée, statuts précaires, manque de consultation, nécessité d'être concentré tout le temps. 
temps, dénigré les médias. Renaudot $\left(17^{\text {ème }}\right.$ siècle) et Zola (19 ${ }^{\text {ème }}$ siècle) exprimaient déjà que le manque d'approfondissement de l'information dans la course contre le temps (Vitalis et al., 2000, p. 18). Dans son ouvrage sur la mythologie des journalistes, Jacques Le Bohec n'évoquet-il pas des vices tels que le manque de moyens humains et temporels accordés aux journalistes pour qu'ils puissent prendre du recul et vérifier correctement leurs informations (1998, p. 320) ? Et Cyril Lemieux (2000) ne consacre-t-il pas un chapitre entier à la thématique du suivisme?

Lors d'une conférence réalisée à Bruxelles dans le cadre des États généraux des médias d'information (2012), Denis Ruellan traite lui aussi des vices de la profession et des malaises qui en découlent. "Il y a souffrance quand on n'est pas ce qu'on prétend être. Ce qui gêne les journalistes ce n'est pas de ne pas être les seuls producteurs [à la source de leurs articles], mais c'est de le dire » (2011). Cette interprétation nous semble particulièrement éclairante en regard de nos données empiriques.

Les journalistes interrogés critiquent en effet leurs propres impostures. C'est le cas des webjournalistes qui désavouent leur sélection sensationnaliste de l'information ou leur propre manque de vérification de l'information. Parce qu'ils souhaitent être reconnus en tant que véritables journalistes, ils éprouvent un malaise quand ils doivent chaparder une information, copier-coller un texte. Les journalistes traditionnels, eux, peuvent se sentir 'imposteurs' quand on leur demande de pratiquer un travail multi-supports qu'ils ne maitrisent pas complètement.

\section{Imbrication des représentations positives et négatives}

La critique semble omniprésente sur notre terrain d'observation. Toutefois, il convient de prendre quelques précautions. En effet, les discours ont été recueillis dans le cadre d'entretiens semi-directifs, laissant une certaine liberté à l'interviewé. On peut supposer que les journalistes interrogés ont perçu, à tort ou à raison, certains objectifs de l'enquêteur (dénoncer les vices du journalisme en ligne par exemple). Ceci a sans aucun doute pu les inciter à développer un discours plus critique, plus négatif à l'égard du webjournalisme. Nous pourrions même imaginer que certains journalistes nous ont instrumentalisée en dénonçant à outrance leurs conditions professionnelles pour tenter de les améliorer. Alors certes, la critique est bien présente dans les 
discours d'acteurs. Mais, en regard des précautions énoncées ci-avant, les discours positifs prennent également une importance nouvelle.

Il est d'ailleurs frappant de constater que plusieurs aspects négatifs énoncés possèdent un revers, une facette positive. Par exemple, alors qu'un journaliste trouve les horaires du Web difficiles, un autre les apprécie. D'aucuns apprécient jongler avec les différentes potentialités du support, alors que la perspective d'un travail multi-support est très démotivante aux yeux d'autres journalistes. Certains aiment la créativité qu'ils peuvent développer sur le Net, alors que d'autres jugent leurs tâches trop techniques et trop répétitives. Dans certaines rédactions, les journalistes se plaindront des statuts précaires des équipes Web, alors que, par ailleurs, la stabilité des statuts sera un argument pour pousser certains journalistes sur le Net. Certains trouveront les relations tendues dans une rédaction Web, alors que la bonne ambiance au sein de l'équipe est vantée dans d'autres lieux.

En poussant plus avant cette analyse, nous remarquons d'ailleurs que ce sont parfois les mêmes personnes qui mentionnent un atout du Web et le défaut qui y est associé. Ainsi, la relation renouvelée à l'internaute est appréciée en même temps que les modalités de gestion de cette interactivité sont souvent critiquées. L'appréciation ou la dépréciation d'un aspect semble ici dépendre de contingences locales ou individuelles. Mais prenons un autre exemple : il n'est pas contradictoire d'apprécier de traiter l'actualité chaude dans l'instantané et de constater en même temps que ce travail en flux tendu est épuisant, qu'il ne permet jamais d'approfondir un sujet, et qu'il peut déboucher sur un travail bâclé, voire sur un manque de vérification. Dans ce cas précis, on pourrait penser que les webjournalistes qui travaillent en flux tendu légitiment, par leur simple pratique quotidienne, un système dont ils sont par ailleurs otages.

Les discours positifs et négatifs sont donc pleinement imbriqués. La critique, très acerbe à première vue, ne peut être désolidarisée d'un ensemble de discours qui la temporisent, continuellement. D'ailleurs, il serait méthodologiquement illusoire de nous en tenir aux deux tableaux ci-dessus qui synthétisent la réalité de façon un peu artificielle, pour mieux la mettre en ordre. Ces tableaux ne sont finalement constitués que d'extraits d'entretiens qui doivent être mis en relation avec d'autres moments desdits entretiens. Par exemple, nous avons demandé aux journalistes quels sont les atouts qu'ils pourraient tirer de leur pratique du journalisme en ligne (ou de leur collaboration avec des 
webjournalistes). Les réponses à cette question viennent, d'une certaine manière, compléter le panorama des aspects positifs énoncés à l'égard du webjournalisme.

$\mathrm{Si}$ on synthétise les arguments énoncés à cet égard (Degand, 2012, pp. 408-411), on constate en premier lieu que le webjournalisme invite les journalistes à repenser leur production et à en améliorer la qualité. Parce qu'Internet oblige les médias traditionnels à repenser leurs contenus. Mais aussi parce que la présence de blogueurs oblige les journalistes à trouver d'autres angles, à faire valoir leur regard journalistique. " Ça nous oblige à être meilleurs » (J. presse imprimée, Sudpresse, Décembre 2009). Deuxièmement, le site Web permet aussi au journaliste de mieux rentabiliser et valoriser son travail, lorsqu'il profite de la capacité d'archivage du site ou de l'espace illimité de la toile. Troisièmement, Internet a renouvelé la relation au public. Très concrètement, pour le journaliste, Internet permet d'avoir accès à un public plus large, d'en connaître de façon plus détaillée les habitudes de consommation et les réactions. Quant aux journalistes qui collaborent effectivement avec les équipes Web, ils engrangent des apprentissages techniques (utilisation des hyperliens, des mini-caméras, des logiciels de modération, des chats, etc.) et ils accèdent à une compréhension plus fine des logiques de leur profession, dans sa globalité, depuis les choix d'édition jusqu'au contraintes qui pèsent sur l'entreprise. Ces éléments recoupent bien entendu les résultats d'autres enquêtes qualitatives (Francoeur, 2012, pp. 74-76). Et si l'on s'en tenait à ce point de vue centré sur les atouts du journalisme en ligne, l'intégration du Web dans les rédactions traditionnelles semblerait, in fine, et malgré toutes les critiques énoncées ci-avant, hautement bénéfique pour les professionnels impliqués.

\section{Stratégies discursives}

En fin de compte, les journalistes interrogés semblent être tiraillés entre, d'une part, la nécessité de dévoiler les faiblesses du journalisme en ligne tel qu'il est organisé dans certaines rédactions et, d'autre part, leur volonté de défendre les journalistes en ligne (qu'ils sont ou qu'ils côtoient), ces journalistes appartenant au pôle dominé de la profession et qui pourtant travaillent dur aux côtés de collègues qui, parfois, les déconsidèrent. Selon que les personnes interrogées souhaitent plutôt insister sur un aspect ou l'autre de la réalité qu'ils vivent, ils développeront un discours de dépréciation ou de valorisation 
du journalisme Web. Les discours, en l'occurrence, relèvent donc bien de stratégies discursives.

Florence Le Cam avait déjà utilisé ce concept de stratégies, qu'elle emprunte notamment à Anselm Strauss (1992). Les formes identitaires s'incarnent alors dans la production de stratégies discursives «destinées à réduire l'écart entre l'attribution et l'incorporation des traits identitaires » (Le Cam, 2005, p. 28). Pour l'auteur, «Ces stratégies relèvent de trois intentions : elles visent à la construction, la défense et la pérennisation de la forme identitaire du groupe » (Le Cam, 2005, p. 375).

Dans les discours que nous avons analysés, nous avons notamment repéré des stratégies discursives antagonistes, visant simultanément à défendre l'identité journalistique dans son ensemble et à la faire évoluer, à construire une identité professionnelle nouvelle incluant les caractéristiques du webjournalisme.

Ainsi, les discours dépréciatifs illustreront particulièrement bien les stratégies discursives de défense du groupe. Ces discours sont souvent dirigés par une volonté de maintenir la réputation de la profession, le prestige qu'elle tire de pratiques dirigées vers un journalisme de qualité et qui apparaissent comme menacées dans la configuration actuelle.

Le groupe doit défendre son appellation, son territoire, ses frontières, son expertise, etc. ; toutes ces choses qui semblent constituer sa forme identitaire. Mais la défense repose sur la perception d'une menace, en provenance de l'extérieur. Que ce soit des actions en provenance de l'ensemble 'publics, sources, pairs et institutions' ou bien des nouveaux venus qui tentent de pénétrer sur le territoire, ou encore des tendances d'ordre plus général (économique, politique ou social), ces facteurs entraînent des mécanismes de protection (Le Cam, 2005, p. 86).

On pourrait même voir dans ces discours, parfois assez conservateurs, une tentative de pérennisation de la forme identitaire du groupe, une volonté de statu quo, voire de retour en arrière.

Et je vois cette rédaction qui ne ressemble plus à la rédaction que nous avions dans l'ancien bâtiment où nous avions une vrai rédaction un peu bordélique, anarchique, ou il y avait beaucoup de débats. Et ici, en peu de temps, et je pense vraiment que le web a accéléré ça, cette conception du multimédia, de cette nécessité 
d'être polyvalent... a vu apparaître de nouveaux acteurs dans le métier, qui ne partagent plus ni la ferveur ni la passion des journalistes. [...] Donc cette nécessité pour le journaliste d'accomplir des tâches techniques le limite dans sa vocation première qui est celle de réfléchir (Journaliste Télévision, RTL, Septembre 2009)

Cela dit, les discours de dépréciation ne vont pas forcément de pair avec un désintérêt vis-à-vis du webjournalisme. Certains de ces discours sont d'ailleurs prononcés par des journalistes Web eux-mêmes (auto-critique) qui indiquent, entre autres, les dérives potentielles de leurs propres pratiques. Un enjeu stratégique apparaît en filigrane : une réflexion collective sur les pratiques et les investissements apparaît nécessaire. Aux yeux des acteurs les plus critiques, c'est la qualité du journalisme digital qui est en jeu. Il s'agit bien, pour eux, de dénoncer pour défendre les intérêts de la profession.

Quant aux discours qui valorisent le journalisme Web, ils contribuent à construire une identité élargie du groupe professionnel. Ils cherchent notamment à signaler une certaine qualité de vie et d'emploi accessible aux webjournalistes, mais aussi des qualités personnelles et professionnelles dont ils doivent faire preuve pour mener à bien leurs missions. Ce faisant, les journalistes cherchent à la fois à légitimer leur position en tant qu'acteurs dignes du titre de 'journalistes' et à justifier leur engagement dans leur profession.

\section{Conclusion}

Quand les journalistes expriment ce qui leur plaît ou ce qui leur déplaît dans le webjournalisme, ils posent un acte de nature stratégique. Par leurs discours et leurs arguments, ils tentent symboliquement d'agir sur la réalité qu'ils vivent. Ce processus est sans doute souvent inconscient. Mais il est riche d'enseignements pour le chercheur :

... les stratégies d'acteurs qui, loin d'être neutres, dévoilent : des représentations particulières du monde, - des intentionnalités spécifiques qui visent à produire du sens à partir de l'observation ou de la réalisation d'une pratique sociale spécifique, - des intentionnalités spécifiques qui visent à se positionner dans un contexte particulier de relations d'acteurs, donc constamment à gérer les relations aux autres, - la construction d'un rôle 
personnel particulier, - les tentatives d'asseoir, d'orienter, de défendre ou de faire la promotion d'une activité particulière (Lecam, 2010, p . 13).

En s'appuyant sur les travaux de Sophie Moirand et de Mikhaïl Bakhtine, Florence Le Cam insiste sur le caractère dialogique de tout discours : les discours se répondent les uns aux autres. Dans cette perspective, nos entretiens ne sont plus que le fragment d'une réflexion globale, d'une grande discussion sans fin que les journalistes alimentent à la fois individuellement et collectivement.

Notre recherche particulière aura montré que les discours journalistiques se déploient selon un schéma cyclique dans lequel la critique à l'égard du webjournalisme est sans cesse temporisée par des arguments plus positifs. C'est ce qui permet à la critique de se renouveler, d'évoluer.

La critique pourrait alors être salutaire pour le journalisme. Si l'on se penche sur les écrits de Luc Boltanski et d'Eve Chiapello (1999), nous verrons que ces auteurs se sont également intéressés au rôle de la critique. Ils ont montré qu'elle avait été essentielle au maintien du capitalisme, au fil des décennies. Le capitalisme serait parvenu à incorporer les valeurs au nom desquelles il était critiqué et par là même à désarmer ses propres critiques. Le journalisme, sans être en rien un objet comparable au capitalisme, semble partager avec lui, cette malléabilité, cette faculté de se transformer.

La critique, quand elle est contrebalancée de discours plus positifs, aurait alors une incidence reconfigurante sur le journalisme dans son ensemble, et notamment sur ses composantes identitaires. Les journalistes Web vont, d'une certaine manière, intégrer les critiques qui sont formulées à leur égard, les assumer et entamer une reconstruction identitaire sur la base de nouvelles valeurs, de nouvelles compétences, de nouvelles pratiques professionnelles. Sur notre terrain d'observation, nous avons par exemple pu rencontrer des webjournalistes qui assumaient le fait qu'ils ne font pratiquement pas de 'terrain', peu d'interviews et qu'ils recomposent les productions d'autres plutôt que d'écrire véritablement de nouveaux articles :

On n'est plus sur le terrain, on perd sa signature [...] Pour l'ego, c'est très dur, mais je trouve que c'est une belle leçon d'humilité parce que ça t'oblige à faire repasser l'info avant ta personne. [...] Mais c'est dur au début de perdre une partie 
de son identité qu'on croit être dans sa signature [...] Donc là aussi, il y a une humilité à avoir... C'est une personne qui est sur le terrain et qui transmet [l'info]. Donc je crois qu'il faut bien respecter ces sources-là (Journaliste Web, Le Soir, Juin 2009).

Dans notre exemple, le journaliste évoque explicitement une 'perte' d'identité, qu'il a vécue lors de son passage du papier au Web. Il fait preuve par ailleurs d'une incorporation des critiques (celle relative au manque de terrain en l'occurrence). Et il y donne réponse. Ce faisant, il revendique une nouvelle « identité pour soi (Dubar, 2000, p. 4). De la même manière, quand les journalistes critiquent le webjournalisme, ils pointent l'altérité, ce qu'ils ne veulent pas être ou ce qu'ils sont malgré eux. Ce type de réflexion illustre une perpétuelle (re)construction identitaire qui prend corps, petit à petit, au fil d'un dialogue constant.

Les stratégies discursives analysées dans cet article reflètent donc des formes identitaires relatives au (web)journalisme en continuelle recomposition. La critique (et sa temporisation) permet dès lors aux journalistes de se repenser, de réaffirmer et de réévaluer constamment les raisons de leur engagement dans la profession journalistique.

\section{Références}

Bernier, M.-F. (2008). Journalistes au pays de la convergence, Sérénité, Malaise et détresse dans la profession. Québec : Presses de l'Université de Laval.

Bettels, T. (2005). Newsdesk' und 'crossmedia'. Eine Analyse innovativ arbeitender Zeitungsredaktionen in Europa am Beispiel der 'Main Post' in Deutschland und 'Nordjyske Medier' in Danemark, Dissertation doctorale, University of Applied Sciences, Darmstadt cité par Meier, K. (2007). Innovations in Central European Newsrooms, Journalism practice, 1(1), 4-19.

Boczkowski, P. (2009). Technology, Monitoring and Imitation in Contemporary News Work, in Communication, Culture \& Critique, 2, 39-58.

Boltanski, L., \& Chiapello, E. (1999). Le nouvel esprit du capitalisme, Paris : Gallimard. Carvajal, M., \& Garcia Aviles, J. A. (2008). Integrated and Cross-Media Newsroom Convergence, Two Models of Multimedia News Production - The Cases of Novotécnica and La Verdad Multimedia in Spain, Convergence, 14(2), 221-239.

Degand, A. (2012). Le Journalisme face au Web : Reconfiguration des pratiques et des représentations professionnelles dans les rédactions belges francophones, Dissertation doctorale. Presses Universitaires de Louvain, Louvain-la-Neuve, Disponible à : http://dial.academielouvain.be.

Deleu, C., Demers, F., \& Paradis, M. (1998). Internet, les médias et les journalistes : les 
expériences nord-américaines et françaises, Les Cahiers Média, 4. Québec.

Deuze, M., \& Fortunati, L. (2011). Journalism Without Journalists, On the Power Shift from Journalists to Employers and Audiences. Dans Meikle, G., \& Redden, G., News online, Transformations and Continuities. Grande Bretagne : Palgrave Mc Millan, 164-177.

Domingo, D., Masip, P., \& Mico, J. L. (2008). Jumping on the Bandwagon: Innovations Discourses and Practices in the Catalan Public Broadcasting Corporation, Barcelona, ECREA II Conference.

Domingo, D., \& Paterson, C. (Ed.) (2008). Making Online News, the Ethnography of New Media Production. Peter Lang Publishing Group.

Dubar, C. (2000). La crise des identités: l'interprétation d'une mutation, Paris : PUF.

Erdal, I. J. (2009). Cross-media (re)production cultures, Convergence, 15(2), 215-231.

Estienne, Y. (2007). Le journalisme après Internet, Paris : L'Harmattan.

Fenton, N.J, \& Witschge, T., (2011) 'Comment is Free, Facts are Sacred': Journalistic Ethics in a Changing Mediascape. Dans Meikle, G., \& Redden, G., News Online, Transformations and Continuities. Grande Bretagne : Palgrave Mc Millan, 148-163.

Fion, C. (2008). Le moral et le jugement des journalistes sur leur métier et leur profession, en Belgique francophone, Dissertation de fin d'études. Louvain-laNeuve : UCL.

Francoeur, C. (2012). La transformation du service de l'information de Radio-Canada, Québec : Presses de l'Université du Québec.

Friend, C., \& Singer, J. B. (2007). Online Journalism Ethics: Tradition and Transition. New York: Armonk.

Garcia, E. P. (2008). Print and Online Newsrooms in Argentinean Media: Autonomy and Professional Identity. Dans Domingo, D., \& Paterson, C. (eds), Making Online News, the Ethnography of New Media Production. New York : Peter Lang, 61-76.

Grevisse, B. (2010). Déontologie du journalisme, Enjeux éthiques et identités professionnelles. Bruxelles : De Boeck.

Huang, E., Rademakers, L., Fayemiwo, M. A., \& Dunlap, L. (2004). Uncovering the Quality of Converged Journalism, A Case Study of The Tampa Tribune News Stories, Convergence, 10(4). Disponible à : http://www.poynter.org/resource/69008/ USF_study1.pdf.

Kovach, B., \& Rosenstiel, T. (2001). The Elements of Journalism. New York: Crown Publishers.

Le Cam, F. (2010). Histoires et filiations du terme 'Weblog' (1992-2003). Perspectives pour penser l'histoire de certaines pratiques sociales sur le Web, Les enjeux de l'information et de la communication, Gresec, 97-120.

Le Cam, F. (2005). L'identité du groupe des journalistes du Québec au défi d'Internet, Dissertation doctorale, Université de Laval et Université Rennes 1, Disponible à : http://tel.archives-ouvertes.fr/tel-00011013/en/

Lemieux, C. (2000). Mauvaise presse. Une sociologie compréhensive du travail journalistique et de ses critiques. Paris : Métailié.

Micó, J., Masip, P., \& Barbosa, S. (2009). Models of Business Convergence in the Information Industry: A Mapping of Cases in Brazil and Spain, Brazilian Journalism research, 5(1).

Paillé, P., \& Mucchielli, A. (2003). L'analyse qualitative en sciences humaines et sociales, Paris : Armand Colin.

Palacios, M., \& Diaz-Noci, J. (Eds) (2009). Online Journalism: Research Methods. 
A Multidisciplinary Approach in Comparative Perspective. Argitalpen Zerbitzua, Universitad del País Vasco, Euskal Herriko Uniberstsitatea. Disponible à : www. argitalpenak.ehu.es.

Pavlik, J., \& Ross, S. (2000). Journalism Online: Exploring the Impact of New Media on News and Society. Dans Albarran, A. B., \& Goff, D. H., Understanding the Web. Ames, Iowa: Iowa State University Press.

Pélissier, N. (2003). Un cyberjournalisme qui se cherche, Hermès, 35, Paris.

Quandt, T. (2008). News Tuning and Content Management: An Observation Study of Old and New Routines in German Online Newsrooms. Dans Paterson, C. ,\& Domingo, D., (eds.), Making Online News, The Ethnography of New Media Production. New York: Peter Lang, 77-97.

Ringoot, R., \& Utard, J.-M. (2005), Le journalisme en invention, Nouvelles pratiques, nouveaux acteurs. Rennes : Presses Universitaires de Rennes.

Singer, J. B. (2004). Strange Bedfellows ? Diffusion of Convergence in Four News Organisations, Journalism Studies, Cardiff, 5(1), 3-18.

Strauss, A. (1992), La trame de la négociation. Sociologie qualitative et interactionnisme, Paris : L'Harmattan.

Vitalis, A., Tétu, J.-F., Palmer, M., \& Castagna, B. (dirs.) (2000). Médias, temporalités et démocratie. Rennes : Apogée/PUF. 